\title{
Autocoloscopie à Chäsitz
}

\section{Rolf H. Adler}

Prof. ém., Dr méd., ancien médecin-chef du service de médecine interne, C.-L.-Lory-Haus, Inselspital Bern

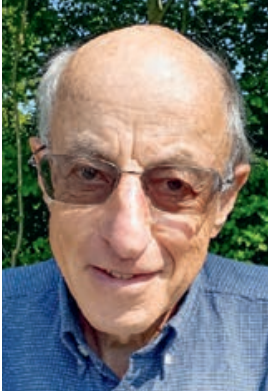

Le SonntagsZeitung du 22 juillet 2018 consacre un article à la télémédecine et à ses avantages. Depuis le $1^{\mathrm{er}}$ août, à Chäsitz, il est possible de se faire soi-même une coloscopie. Le SonntagsZeitung ne peut pas encore en parler, bien sûr. A la pharmacie Chabisreutinger, le pharmacien, M. Bizarre, a aménagé une salle pour les coloscopies. L'examen coûte CHF 345 et est pris en charge par la caisse-maladie DO IT YOURSELF.

Vendredi, Ivo Gastroactif a avalé deux litres de jus de pomme, et un autre litre deux heures avant le début de la coloscopie, le samedi. Il s'allonge, on lui remet un casque et on fixe un micro à l'avant de sa chemise. Il va être mis en liaison avec le Dr Passageenforce, de l'Inselspital. Un objet en forme de serpent pend du plafond: le coloscope. M. Gastroactif étant droitier, il est prié de prendre le bout du coloscope, de s'allonger sur le côté gauche et d'introduire l'appareil (l'installation n'est pas encore disponible pour les gauchers). Cela lui fait mal et il n'y arrive pas. Le Dr Passageenforce lui recommande de retenir sa respiration et de pousser, comme aux toilettes. Ca y est. Au bout de $35 \mathrm{~cm}$, Ivo est prié de se tourner sur le dos. Une douleur fulgurante le traverse. Le Dr Passageenforce annonce un olympe dans le champ visuel. Ivo demande des explications. Il n'a jamais entendu parler d'un olympe dans le gros

\section{Depuis le $1^{\text {er }}$ août, à Chäsitz, il est possible de se faire soi-même une coloscopie.}

intestin. Le Dr Passageenforce corrige, ce qu'il voit est un polype. Il demande à Ivo de bien vouloir introduire davantage l'appareil (jusqu'à la valve iléocaecale, mais le patient ne peut pas comprendre cela bien sûr). Les douleurs sont devenues intenses. Il peut maintenant retirer doucement l'instrument.
Ivo se lève et se rend dans l'espace de vente de la pharmacie. Là, il tombe sur une affiche: «La joie dans le cœur et les oignons dans l'estomac: paix et flatulences garanties.» Le Dr Bizarre lui vend des garnitures, au cas

Le dimanche les douleurs abdominales deviennent si violentes que son médecin de famille l'envoie en taxi aux urgences.

où il saignerait de l'anus. Ivo remonte le Stotzgrotzen jusque chez lui en voiture, à maximum $30 \mathrm{~km} / \mathrm{h}$, la limitation de vitesse ayant enfin été adoptée par le Conseil communal pour protéger les chats.

Le dimanche, en lisant le SonntagsZeitung, les douleurs abdominales deviennent si violentes qu'il appelle son médecin de famille, le Dr Gestiondeségoûts, qui l'envoie en taxi aux urgences de l'Inselspital. Là, Ivo subit une laparotomie pour réparer une perforation du gros intestin. La facture s'élève maintenant à CHF 8450.

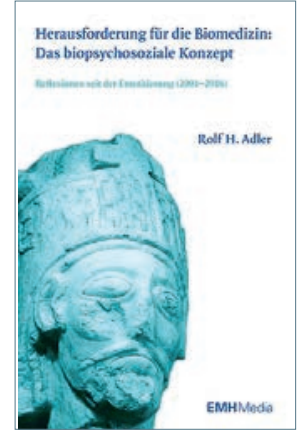

Pour lire une évaluation un peu moins terrible de la télémédecine, se reporter à l'article sur les dangers de la télémédecine, intitulé «Telemedizin gefährlich, unwirtschaftlich und entbehrlich" (en allemand, p. 89-92) et signé du même auteur.

Rolf H. Adler

Herausforderung für die Biomedizin: Das biopsychosoziale Konzept. Basel: EMH-Verlag; 2017. 171 Seiten. 24.50 CHF ISBN 978-3-03754-107-4. 